\title{
Identification of putative fecundity-related gustatory receptor genes in the brown planthopper Nilaparvata lugens
}

\author{
Kui Kang ${ }^{1 \dagger}$, Pan Yang ${ }^{1 \dagger}$, Li-E Chen ${ }^{1}$, Rui Pang ${ }^{1}$, Lu-Jun Yu', Wen-Wu Zhou'², Zeng-Rong Zhu ${ }^{2}$ and \\ Wen-Qing Zhang ${ }^{* *}$ (1)
}

\begin{abstract}
Background: The insect gustatory system plays a central role in the regulation of multiple physiological behaviors and the co-evolution between insects and their hosts. The gustatory receptors (Gr) are important to allow insects to sense their environment. It is critical to the selection of foods, mates and oviposition sites of insects. In this study, the Gr family genes of the brown planthopper (BPH) Nilaparvata lugens Stål (Hemiptera: Delphacidae) were identified and analyzed, and their potential relationship to the fecundity of BPH was explored by RNA interference (RNAi).

Results: We identified 32 putative Gr genes by analyzing transcriptome and genome data from BPH. Most of these Gr proteins have the typical structure of seven transmembrane domains. The BPH Gr genes (N/Grs) were expressed in virtually all tissues and stages, whilst higher transcript accumulations were found in adult stages and in the midguts of females. Based on the phylogenic analysis, we classified NIGrs into five potential categories, including 2 sugar receptors, 2 Gr43a-like receptors, $7 \mathrm{CO}_{2}$ receptors, 5 bitter receptors and 13 N/Grs with unknown functions. Moreover, we found that $10 \mathrm{NIGrs}$ have at least two alternative splicing variants, and obtained alternative splicing isoforms of $5 \mathrm{~N} / \mathrm{Gr}$. Finally, RNAi of $29 \mathrm{~N} / \mathrm{Grs}$ showed that 27 of them are related to the transcript levels of two fecundity related genes vitellogenin and vitellogenin receptor.
\end{abstract}

Conclusions: We found $32 \mathrm{Gr}$ genes in BPH, among which at least 27 are required for normal expression of fecundity markers of this insect pest. These findings provide the basis for the functional study of Grs and the exploration of potential genes involved in the monophagous character of $\mathrm{BPH}$.

Keywords: Nilaparvata lugens, Gustatory receptor, Alternative splicing, Fecundity

\section{Background}

Insects interact with their environment primarily through a sensitive chemosensory system that can detect and discriminate a diverse array of chemicals. This system plays critical roles in the survival and reproductive success of insects, mediating their behavioral responses to food, mates, and oviposition sites $[1,2]$. Gustatory receptors (Grs), members of the chemosensory superfamily are mainly distributed in the chemosensory organs. They are responsible for distinguishing $\mathrm{CO}_{2}$ and

\footnotetext{
* Correspondence: Isszwq@mail.sysu.edu.cn

${ }^{\dagger}$ Kui Kang and Pan Yang contributed equally to this work.

'State Key Laboratory of Biocontrol and School of Life Sciences, Sun Yat-sen

University, Guangzhou 510275, Guangdong, China

Full list of author information is available at the end of the article
}

non-volatile chemicals including the nutrients and toxins [3].

Insect Grs, with a typical seven transmembrane structure, were first identified in the Drosophila melanogaster genome based on a bioinformatic approach [4]. Further studies found that there are 68 gustatory receptor proteins in $D$. melanogaster, encoded by 60 gustatory receptor genes through alternative splicing [5-7]. Most gustatory receptor proteins are extraordinarily divergent, sharing only $8-12 \%$ sequence identity at the amino acid level. Some of this divergence could improve the diversity of Grs' responses to ligands [7]. Based on the known ligands to which they respond, Grs were grouped into sugar receptors, $\mathrm{CO}_{2}$ receptors, Gr43a-like receptors,

(C) The Author(s). 2018 Open Access This article is distributed under the terms of the Creative Commons Attribution 4.0 International License (http://creativecommons.org/licenses/by/4.0/), which permits unrestricted use, distribution, and 
bitter receptors, sex pheromone receptors, and unknown receptors [8-13]. With the development of genome sequencing in insects, insect $\mathrm{Gr}$ genes are identified in an increasing number of additional species: Anopheles gambiae has $52 \mathrm{Gr}$ genes encoding $76 \mathrm{Gr}$ proteins [14], and Aedes aegypti has $79 \mathrm{Gr}$ genes encoding $114 \mathrm{Gr}$ proteins [15]. Bombyx mori and Tribolium castaneum have 65 and $220 \mathrm{Gr}$ genes, respectively [16, 17]. Helicoverpa armigera showed the second highest number (197) of $\mathrm{Gr}$ genes among all insect species studied [18].

Alternative pre-mRNA splicing greatly expands the proteome diversity within species by creating different combinations of exons from the same genomic loci [19], and has been most extensively studied in D. melanogaster [20]. Among sixty D. melanogaster Grs, three genes have alternatively spliced transcripts. Gr23a and Gr39a encoded two and four predicted proteins, respectively [4], and Gr28b encodes five predicted proteins [7]. Gr39a is a multifunctional receptor set, and alternative splicing is the mechanism for the generation of molecular forms responsible for different functions, such as pheromonal perception and host plant selection [21]. Gr28b.d, one alternatively spliced transcript of Gr28b, encoded a potential warmth sensor (Gr28B(D)) [22]. T. castaneum Gr214 is a massive alternatively spliced locus with 30 alternative long $5^{\prime}$ exons spliced into three shared 3' exons encoding the $C$ terminus [16]. The alternative splicing pattern of gustatory receptor has also been found in other insect species, such as $A$. aegypti [15] and B. mori [17]. However, the function of these alternatively spliced forms remains unclear.

The brown planthopper (BPH) Nilaparvata lugens (Hemiptera: Delphacidae) is one of the most devastating insect pests of rice [23]. Vitellogenin $(V g)$ and vitellogenin receptor $(V g R)$ were always used as molecular markers of fecundity [24].Gustatory receptors may play an important role in the interaction of $\mathrm{BPH}$ and rice by detecting chemical compounds in rice. In this study, we identified $32 \mathrm{Grs}$ in $\mathrm{BPH}$ based on transcriptome and genome data. Then we analyzed the alternative splicing patterns of all $N l G r s$, and found that 10 of them had alternative splicing events; further studies showed that alternative splicing could alter the protein structure of some NlGrs. Finally, we employed the RNA interference (RNAi) experiments and found that $27 \mathrm{NlGrs}$ are required for normal expression of fecundity markers of this insect pest.

\section{Results}

Transcriptome sequencing and sequence assembly

We performed next-generation sequencing on a cDNA library constructed from the adult head, leg and midgut of $\mathrm{BPH}$. All clean reads were aligned to the reference transcripts (generated in our laboratory, unpublished) and genome [25] of $\mathrm{BPH}$, and identified approximately 47,000 genes with an N50 length of $2300 \mathrm{bp}$ (see Additional file 1: Table S1). The mapping rate was approximately $75 \%$, except in the midgut (57.9\%), which contains a large number of enteric microorganisms (see Additional file 1: Table S1).

\section{Annotation and identification of gr genes}

To identify as many gustatory receptors as possible, three strategies were used. Firstly, we obtained 33 and 10 potential $G r$ sequences from the transcriptome data and genome data, respectively, using reciprocal hit of tBLASTn. 36 potential $G r$ sequences were identified after combining these sequences. The length of them was between $216 \mathrm{bp}$ and $3921 \mathrm{bp}$, and most sequences were shorter than 1000 bp (see Additional file 2: Table S2). We then used pfam to build the second structure model of GR, PF02949, PF08395, and PF06151 and searched for the target sequences in the protein database. Using this method, we obtained 20 potential GR protein sequences, and 3 were identified as olfactory receptors via blast in NCBI; 13 were covered with previously potential sequences. After removing the sequence whose predicted protein length are less than 100 amino acids, only one new potential GR sequence was found, XLOC_018226:3-81 (see Additional file 3: Table S3). Finally, using a conserved sequences search, we obtained 21 potential GR protein sequences, all of which were covered with previously sequences. In summary, we obtained 37 potential $\mathrm{Gr}$ sequences using bioinformatic analyses.

To further determine whether these genes are $\mathrm{Gr}$ genes, we used 3' rapid amplification of cDNA ends (3' RACE) and 5' RACE to obtain longer fragments. After sequencing, the result was tested by using BLAST and compared with the database of National Center for Biotechnology Information. The results showed that 6 potential sequences were not Grs and 4 were identical to each other. In conclusion, we identified $27 \mathrm{Grs}$ in BPH. By combining the sequences with 10 previously reported gustatory receptors [25], a total of $32 \mathrm{NlGrs}$ were identified. Detailed information of the identified and cloned Grs in BPH is available (Table 1).

\section{Phylogenetic analysis}

The phylogenetic analysis result revealed that NlGr1-7 are potential members of the $\mathrm{CO}_{2}$ receptor subfamily (Fig. 1); $N l G r 8$ and $N l G r 9$ are potential members of the Gr43a-like (Drosophila) receptor subfamily [13]. NlGr10 and NlGr11 are potential members of the insect sugar receptor subfamily $[26,27]$. NlGr $12-N l G r 14$ are orthologs to the DmGr66a protein which is responsible for the recognition of various bitter compounds [3]. In addition, NlGr15-NlGr16 might belong to the narrowly tuned Grs required for some bitter compounds, as they 
Table 1 Nucleotide, amino acid, scaffold distribution, alternative splice variant and other information for the identified NIGrs

\begin{tabular}{|c|c|c|c|c|c|c|c|}
\hline GENE & TMHMM & TMPred & НMMTOP & $\mathrm{AA}$ & Nucleic acids(bp) & Scaffold & $\begin{array}{l}\text { Predicted alternative } \\
\text { splice variant number }\end{array}$ \\
\hline NIGr1 & 8 & 8 & 8 & 436 & 1554 & KN153269.1 & 0 \\
\hline N/Gr2 & 7 & 8 & 9 & 461 & 1717 & KN153837.1 & 2 \\
\hline N/Gr3 & 7 & 8 & 8 & 440 & 1631 & KN153837.1 & 0 \\
\hline N/Gr4 & 4 & 4 & 4 & 265 & 798 & KN153837.1 & 0 \\
\hline NIGr5 & 6 & 7 & 7 & 425 & 1670 & KN152938.1 & 0 \\
\hline N/Gr6 & 5 & 5 & 5 & 488 & 2188 & KN152682.1 & 10 \\
\hline NIGr7 & 8 & 8 & 8 & 413 & 1605 & KN153186.1 & 0 \\
\hline N/Gr8 & 5 & 6 & 6 & 357 & 2176 & KN153141.1 & 10 \\
\hline NIGr9 & 4 & 6 & 4 & 400 & 3216 & & 10 \\
\hline NIGrio & 7 & 8 & 8 & 471 & 2121 & KN151984.1 & 2 \\
\hline NIGr11 & 7 & 7 & 8 & 466 & 1342 & KN152173.1 & 0 \\
\hline NIGr12 & 6 & 6 & 6 & $339^{a}$ & 1785 & KN152706.1 & 0 \\
\hline NIGr13 & 8 & 8 & 7 & 401 & 1625 & KN152706.1 & 0 \\
\hline NIGr14 & 6 & 6 & 7 & 339 & 1536 & KN152506.1 & 0 \\
\hline N/Gr15 & 4 & 4 & 6 & 321 & 1604 & KN152468.1 & 2 \\
\hline NIGr16 & 4 & 8 & 6 & 383 & 1149 & KN152007.1 & 0 \\
\hline NIGri7 & 7 & 8 & 9 & 520 & 1561 & KN152004.1 & 4 \\
\hline N/Gr18 & 3 & 7 & 6 & 374 & 1308 & KN153751.1 & 3 \\
\hline NIGr19 & 3 & 3 & 3 & $218^{\mathrm{a}}$ & 1730 & KN152376.1 & 4 \\
\hline NIGr2O & 6 & 7 & 7 & 449 & 2062 & KN152775.1 & 2 \\
\hline$N \mid G r 21$ & 7 & 6 & 8 & 352 & 1435 & KN154548.1 & 0 \\
\hline NIGr22 & 6 & 6 & 5 & 370 & 1110 & KN154035.1 & 0 \\
\hline NIGr23 & 7 & 7 & 6 & 398 & 1537 & KN153338.1 & 2 \\
\hline NIGr24 & 7 & 7 & 7 & 379 & 1141 & KN152091.1 & 0 \\
\hline NIGr25 & 3 & 3 & 3 & 218 & 1580 & KN152147.1 & 0 \\
\hline NIGr26 & 5 & 5 & 5 & 429 & 1750 & KN152989.1 & 3 \\
\hline N/Gr27 & 0 & 0 & 0 & $0^{\mathrm{b}}$ & 212 & & 0 \\
\hline$N / G r 28$ & 6 & 6 & 6 & 357 & 1071 & KN154570.1 & 2 \\
\hline N/Gr29 & 6 & 6 & 6 & 373 & 2032 & KN152059.1 & 0 \\
\hline NIGr30 & 0 & 0 & 0 & $0^{\mathrm{b}}$ & 416 & & 0 \\
\hline N/Gr31 & 3 & 3 & 3 & 153 & 1926 & KN152885.1 & 0 \\
\hline NIGr32 & 0 & 0 & 0 & 131 & 394 & KN154495.1 & 0 \\
\hline
\end{tabular}

${ }^{a}$ Has more than one ORF regions, and the longest ORF was used for protein translation ${ }^{\mathrm{b}} \mathrm{ORF}$ is incomplete

are in the same branch as DmGr93a or DmGr33a [28]. Moreover, NlGr17- NlGr18 have a simple orthologous relationships with DmGr77a and other DmGrs, whilst their functions remain unclear [29]. The remaining 11 NlGrs do not have confident phylogenetic relationships with Grs from other insects (Fig. 1).

\section{Expression profiles of $\mathrm{NIGr}$ genes}

qRT-PCR was used to investigate the expression pattern of the $\mathrm{Gr}$ genes in different tissues and developmental stages of $\mathrm{BPH}$. Across the developmental stages, some
Grs have a higher transcript accumulation in 4th or 5th day old brachypterous female adult than other developmental stages, such as NlGr6, NlGr9, NlGr10, NlGr14, NlGr18, NlGr19, NlGr16 and NlGr25 (Fig. 2). Five Grs, including NlGr1, NlGr12, NlGr15, NlGr17, and NlGr20, have a higher mRNA expression in nymph stages (Fig. 2). In particular, NlGr12 was only detected in nymph stages, and NlGr29 showed the highest expression in 3rd instars nymphs. Moreover, all $N l G r$ genes were found in the heads, legs, midgut, fat body or female ovaries (Fig. 3). $21 \mathrm{NlGr}$ genes were expressed in multiple tissues. For 


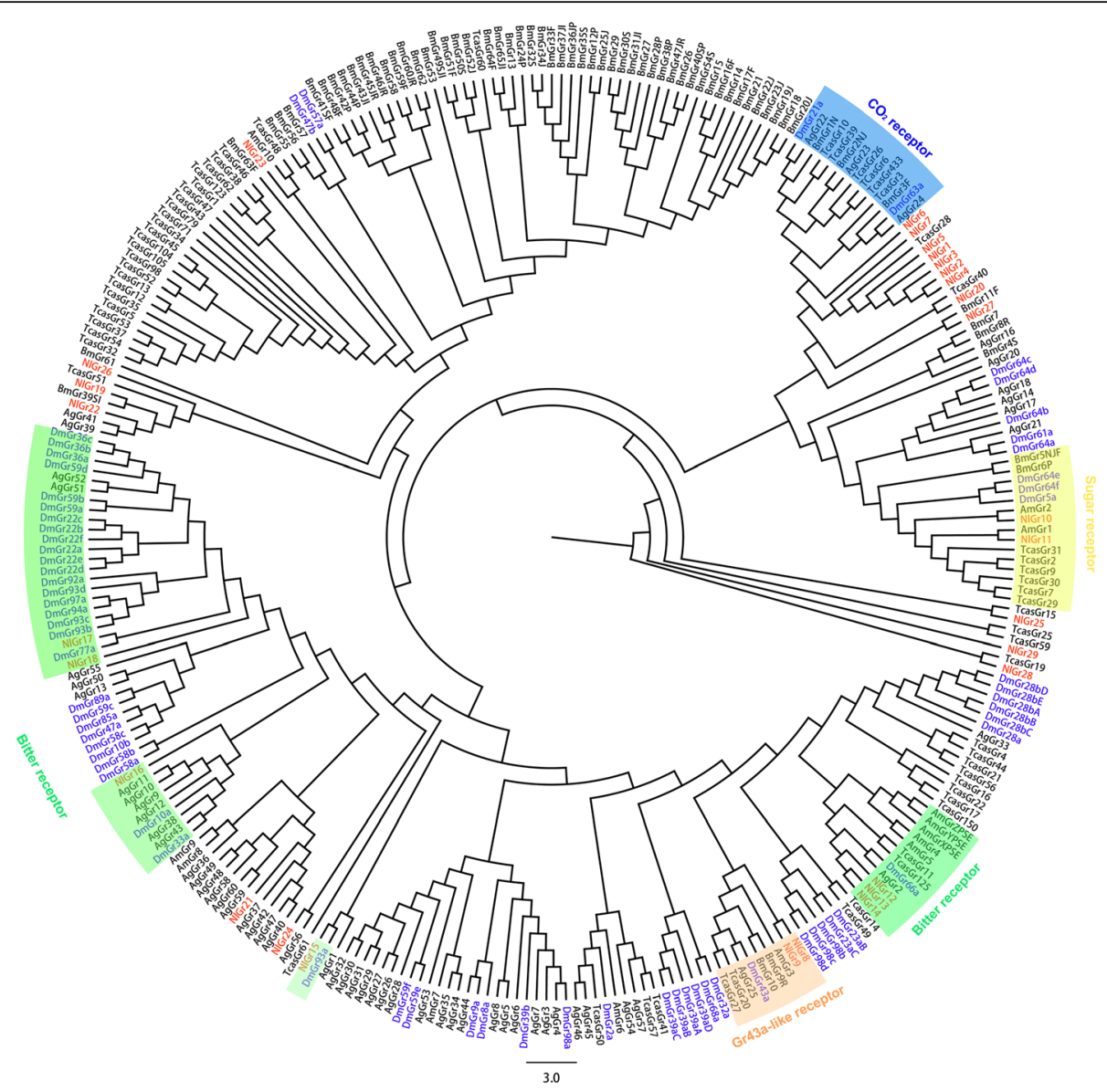

Fig. 1 Phylogenetic relationship of 29 Nilaparvata lugens gustatory receptors (N/Grs) and five published insect gustatory receptors. The reference sequence includes 68 Drosophila melanogaster Grs (DmGrs), 65 Bombyx mori Grs (BmGrs), 13 Apis mellifera Grs (AmGrs), 58 Aedes aegypti Grs (AgGrs), 62 Tribolium castaneum Grs (TcasGrs). DmGrs and N/Grs are highlighted with blue and red letters, respectively. The maximum-likelihood tree was calculated using default settings and the Jones-Taylor-Tornton (JTT) model with partial deletions and 2000 bootstrap replications

example, among them, NlGr24 and NlGr32 were mainly detected in 3 tissues: the midgut, fat body and female ovaries. NlGr16 and NlGr21 were expressed in the midgut and fat body. Conversely, $11 \mathrm{NlGr}$ genes were mainly expressed in specific tissues. For example, NlGr5, NlGr11, NlGr18, NlGr23, NlGr25, NlGr28, NlGr30, and NlGr31 were most highly expressed in the midgut. $N l G r 9$ and $N l G r 14$ were mainly expressed in the fat body. And NlGr15 was mainly expressed in the adult heads (Fig. 3). By analyzing the DEG data, the expression profiles of NlGrs in head, midgut and legs were similar to the qRT-PCR results.

\section{Alternative splicing analysis of NIGrs}

By assembling the transcriptome, a total of 70,361 transcripts were obtained. Among them, 49,930 transcripts were found to contain multiple exons, and 27,880 alternative splicing events were identified. Among the $32 \mathrm{NlGr}$ genes, 13 genes have potential alternative splicing events (Table 1 ). We verified all of the predicted alternative splicing events for NlGrs by PCR, and found that 10 of them had at least two splicing variants (Table 2). By comparison with the genome of $\mathrm{BPH}$, we obtained alternative splicing forms of five NlGrs: NlGr1, NlGr8, NlGr10, NlGr21, and $\mathrm{NlGr} 23$ (Figs. 4 and 5).

NlGr10, a putative sugar receptor, had an alternative first exon event, and was found to have two splicing variants: NlGr10a, which encodes 470 amino acids; and $N l G r 10 b$, which encodes 458 amino acids. NlGr1 and NlGr21 showed cassette exon skipping events, and were found to have two splicing variants, respectively. Another unknown receptor, NlGr23 had an alternative 5' splice site, and the splice site was located in its second exon. NlGr8, with ten exons and ten splicing forms, had the most complex splicing events among all the NlGrs (Fig. 5). The alternative splicing may change the protein structure of these $5 \mathrm{NlGrs.} \mathrm{For} \mathrm{ex-}$ ample, one splicing variants of NlGr23 lost the second and third transmembrane domains, and the NlGr1O splice variants generated by the alternative 


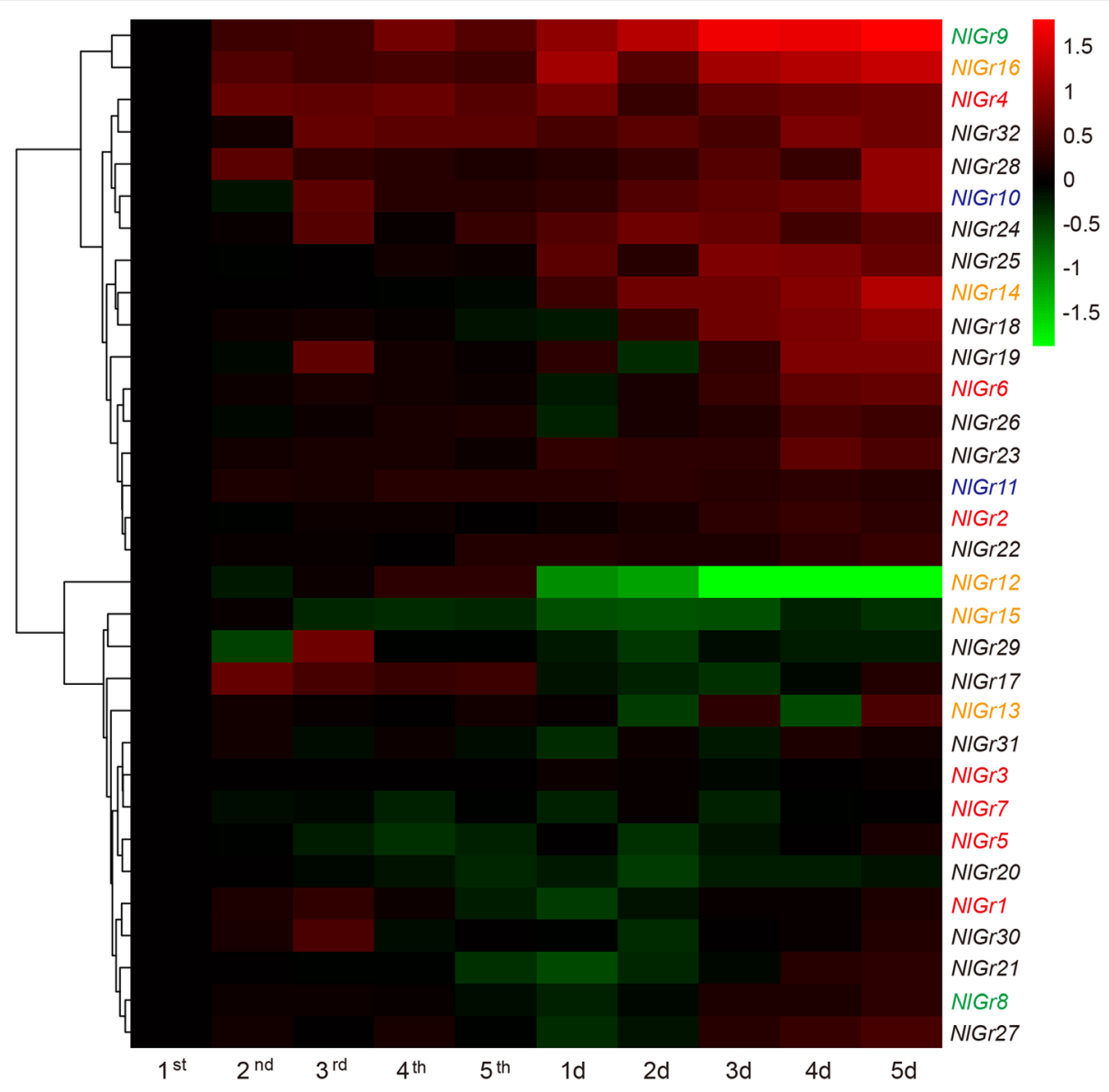

Fig. 2 The expression pattern of NIGrs in different developmental stage. NIGrs expression at various stages including 1st-instar nymph (1st), 2ndinstar nymph (2nd), 3rd-instar nymph (3rd), 4th-instar nymph (4th), 5th-instar nymph (5th), and 1-day- to 5-day-old female adults (1d-5d); mRNA levels of 1st-instar nymphs were used as controls. Different color fonts represent different predicted subfamilies: red: $\mathrm{CO}_{2}$ receptor subfamily; green: Gr43a-like receptor subfamily; blue: sugar receptor subfamily; orange: bitter receptor subfamily; black: unknown functions receptor. All mRNA levels are normalized by the $\beta$-actin mRNA levels, and data are shown as the mean $(n=3)$

first exon and encoded an N-terminal altered NlGR10 protein.

\section{Effects of dsRNA injection on $N / V g$ and $N / V g R$ expression}

To further investigate the function of the $N l G r$ genes in $\mathrm{BPH}, \mathrm{RNAi}$ experiments were performed. Gene silencing of the target genes was achieved by dsRNA injection (Fig. 6a, see Additional file 4: Table S4), except for three NlGrs (NlGr27, NlGr30 and NlGr32), whose nucleotide sequences were less than $450 \mathrm{bp}$ and were too short for primer design. We found three patterns of $N l V g$ and $N l V g R$ mRNA levels in $\mathrm{BPH}$ after silencing the $29 \mathrm{NlGrs}$ (Fig. 6b). In the first type, the transcript of $N l V g$ and $N l V g R$ was decreased when BPH was injected with dsNlGr. The genes with

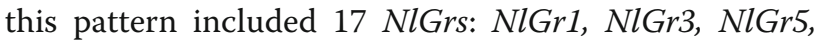
NlGr6, NlGr7, NlGr8, NlGr10, NlGr12, NlGr13, NlGr14, NlGr15, NlGr17, NlGr18, NlGr20, NlGr22, NlGr25, and NlGr29. In the second type, the transcript of $\mathrm{NlVg}$ was increased, while it was decreased for $N l V g R$, when BPH was injected with dsNlGr19, dsNlGr28 or dsNlGr31. And the third type, either $N l V g$ or $N l V g R$ had changed mRNA level after injection. For example, the $N l V g R$ expression was decreased when treated with dsNlGr2, dsNlGr9 or dsNlGr11, whilst no significant change in $N l V g$ mRNA level were found; and the $N l V g R$, but not $N l V g$, showed increased mRNA level when BPH was treated with dsNlGr4 or dsNlGr16 (Fig. 6b). Furthermore, NlGr23 and NlGr26 did not appeare to have significant effect on the expression of $N l V g R$, while the $N l V g$ expression was significantly decreased or increased at $72 \mathrm{~h}$ after with the injection of them, respectively. In summary, except for NlGr21 and NlGr24, the other tested $27 \mathrm{NlGrs}$ affected the transcript accumulation of $N l V g$ or $N l V g R$. These results implied that $N l G r s$ may play an important role in modulating $N l V g$ or $N l V g R$ expression in BPH. Since 


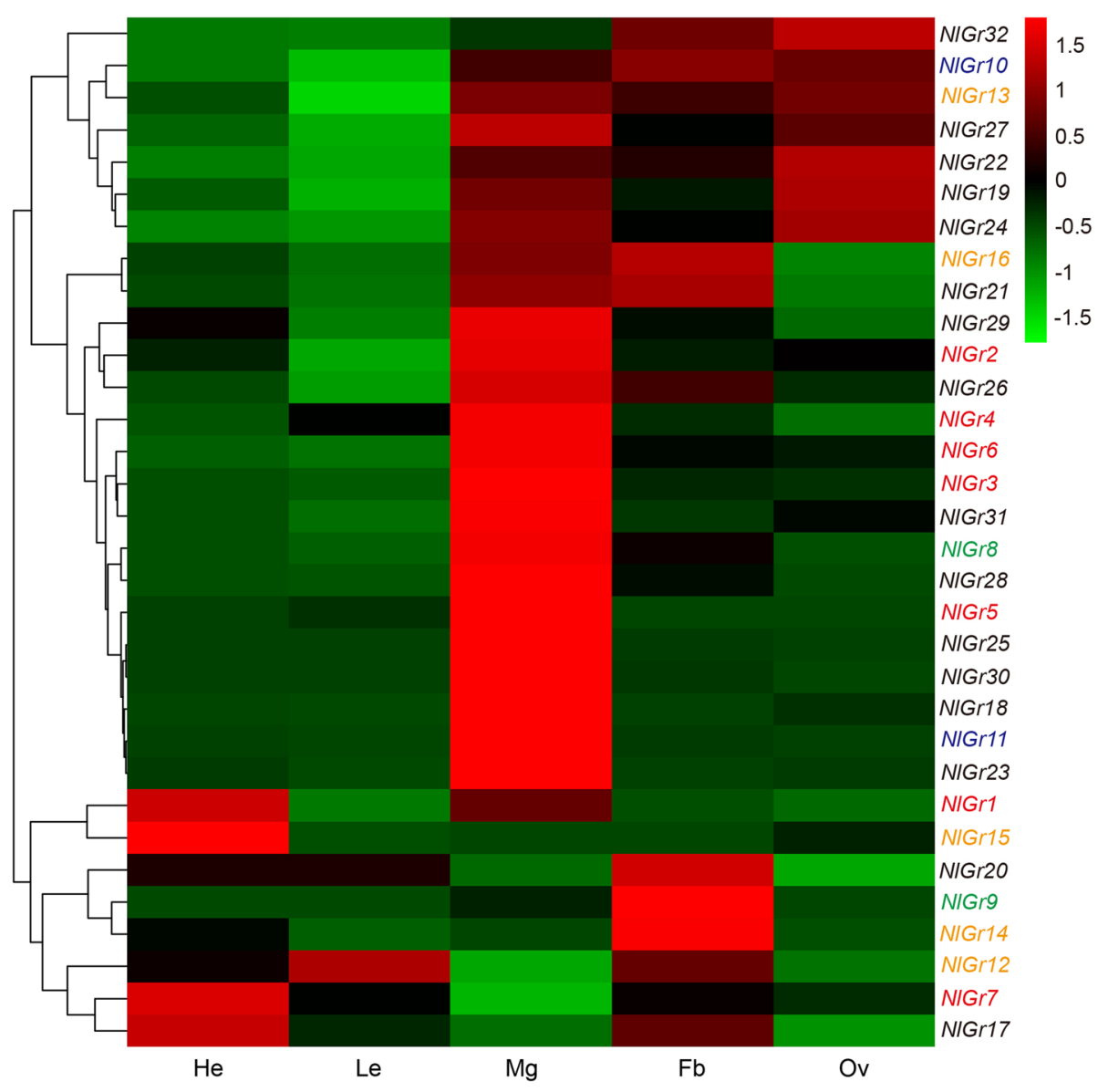

Fig. 3 Tissue specific expression pattern of N/Grs. Expression of N/Grs in various female tissues including the heads (He), legs (Le), midgut (Mg), fat body (Fb) and ovaries (Ov); mRNA levels of legs were used as controls. Different color fonts represent different predicted subfamilies: red: $\mathrm{CO}_{2}$ receptor subfamily; green: Gr43a-like receptor subfamily; blue: sugar receptor subfamily; orange: bitter receptor subfamily; black: unknown functions receptor. All mRNA levels are normalized by the $\beta$-actin mRNA levels. Data are shown as the mean $(n=3)$

Table 2 Clone of alternatively splicing of N/Grs in Nilaparvata lugens

\begin{tabular}{llll}
\hline Gene & Type & $\begin{array}{l}\text { Predicted number of } \\
\text { alternative splice variants }\end{array}$ & $\begin{array}{l}\text { Verified number of } \\
\text { alternative splice variants }\end{array}$ \\
\hline NGG1 & $\begin{array}{l}\mathrm{CO}_{2} \\
\text { receptor }\end{array}$ & 0 & 2 \\
NGr8 & $\begin{array}{l}\text { Gr43a-like } \\
\text { receptor }\end{array}$ & 10 & 10 \\
NGG10 & $\begin{array}{l}\text { Sugar } \\
\text { receptor }\end{array}$ & 2 & 2 \\
NGG12 & $\begin{array}{l}\text { Bitter } \\
\text { receptor }\end{array}$ & 0 & 2 \\
NGr19 & Unknown & 4 & 3 \\
NGG21 & Unknown & 0 & 2 \\
NGG23 & Unknown & 2 & 2 \\
NGG25 & Unknown & 0 & 3 \\
NGr26 & Unknown & 3 & 2 \\
NGr31 & Unknown & 0 & 3 \\
\hline
\end{tabular}

$V g$ and $V g R$ were always used as molecular markers of fecundity [24], it is likely that these $27 \mathrm{NlGrs}$ are required for normal expression of fecundity markers.

\section{NIGr25 response to resistant rice varieties}

qRT-PCR was used to measure the mRNA levels of $\mathrm{NlGr} 25$ from the whole body fed on the sensitive rice variety (TN1) and resistance rice varieties (IR26, IR36, IR56). The results showed that NlGr25 transcript levels were significantly increased at $3 \mathrm{~h}, 6 \mathrm{~h}$ and $12 \mathrm{~h}$ post fed on IR26 compared to fed on TN1. Similar results were observed for IR56. Whereas, feeding on IR36 didn't influence expression of $N l G r 25$ in BPH (Fig. 7).

\section{Discussion}

We identified $32 \mathrm{Gr}$ genes in $\mathrm{BPH}$ by combining transcriptome and genome data [25]. Insect $\mathrm{Gr}$ families appear to be adapted to their ecological niche. For example, the honeybee Apis mellifera has an expansion of the olfactory receptor gene family (163 Ors) and only 


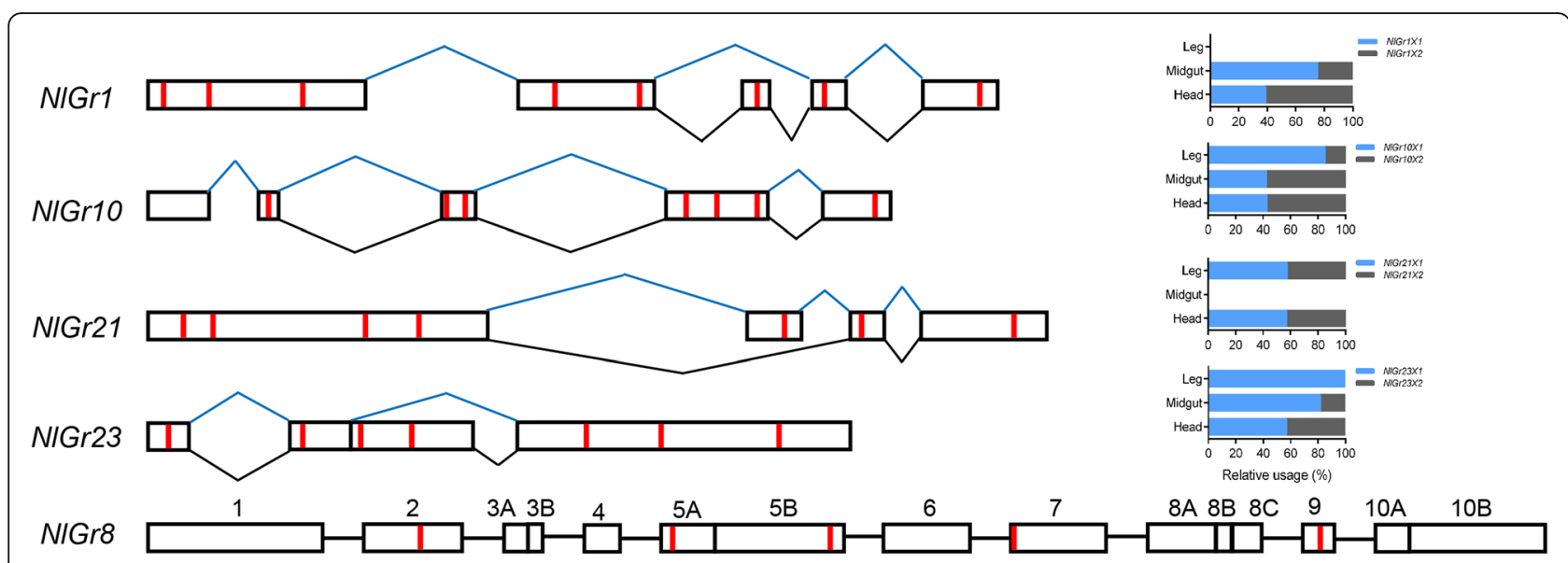

Fig. 4 Alternatively spliced pattern of five NIGrs. The data were verified by PCR with genomic DNA and CDNA as templetes. Bars represent exon sequence; lines represent intron sequences. Blue lines and black lines represent different splicing modes. Red lines in bars represent transmembrance domain. Splicing patterns include cassette exon skipping (N/Gr1, N/Gr21), alternative first exons (N/Gr10), and alternative 5' splice site (NIGr23). The histograms showed the different isoform relative usage from the RNA-seq data, blue bars represent blue line spliceosome

10 Grs [30]. It has been hypothesized that bees have a limited need for Grs in plant secondary metabolite discrimination due to their typical foraging and social behavior [30]. Helicoverpa armigera has three times as many Grs (197) than Ors (64), which may be linked to this species' capacity as a successful generalist, as the expansion presumably broadens the range of plant secondary metabolites detected by this species [18]. The number of NlGr was less than that in the aphid Acyrthosiphon pisum (72 Grs) [31], which is also a hemipteran insect. This may be because BPH is a monophagous insect that only feeds on rice, possibly explaining the reduced number of $\mathrm{Gr}$ genes. A. pisum is a type of omnivorous agricultural pest, and more Grs may allow it to recognize more secondary or primary metabolites from different host plants. Based on phylogenetic analysis, we classified $29 \mathrm{NlGrs}$ into five categories: sugar receptors, Gr43a-like receptors, $\mathrm{CO}_{2}$ receptors, bitter receptors and Grs with unknown functions. No loss of $\mathrm{NlGr}$ function shows that BPH Grs may be able to detect and discriminate large amount of metabolites in rice. Rapid changes in the "bitter" receptor repertoire have been described in Drosophila flies, apparently concomitant with changes in their plant hosts [32].

Transcriptome and qRT-PCR approaches were used to analyze the expression profile of $\mathrm{NlGr}$ genes in different tissues and at different stages, and the results showed that NlGrs was expressed almost in all tissues and stages.

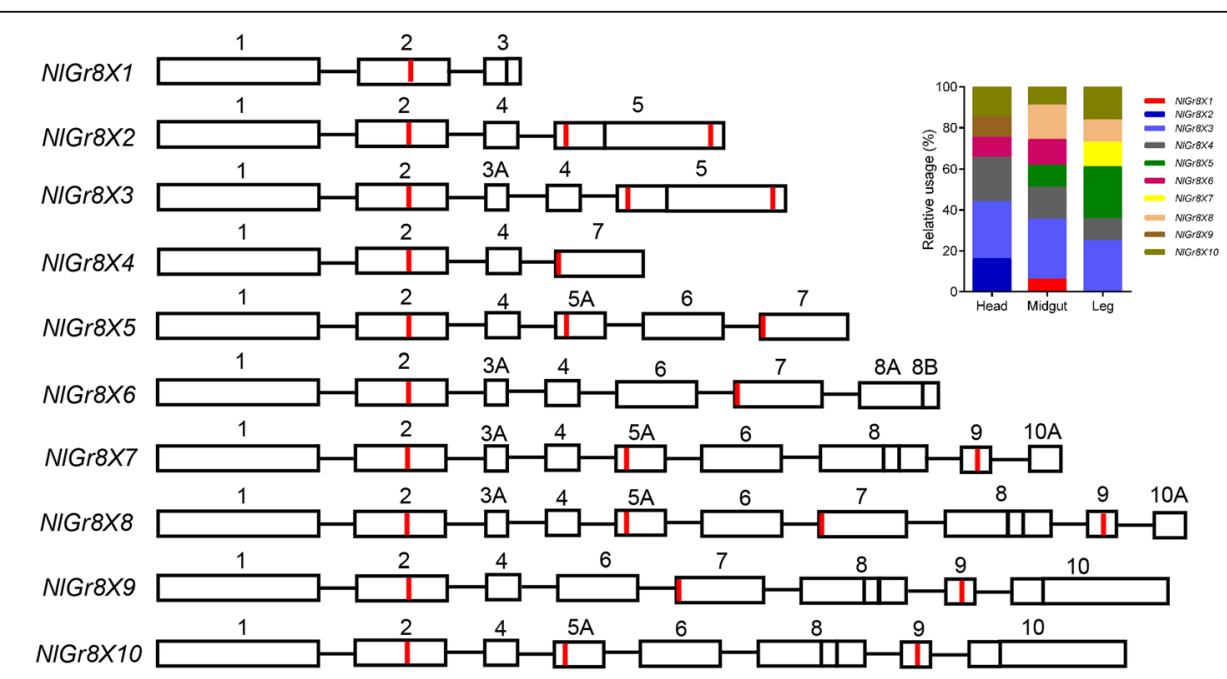

Fig. 5 Ten alternatively spliced patterns of NIGr8. Bars represent exon sequence, and lines represent intron sequence. The data were verified by PCR with genomic DNA and CDNA. Red lines in bars represent transmembrance domain. Splicing patterns include cassette exon skipping, alternative $3^{\prime}$ splice site, alternative last exons and mutually exclusive cassettes. The histograms showed the different isoform relative usage from the RNA-seq data, different color bars represent different spliceosome 
A

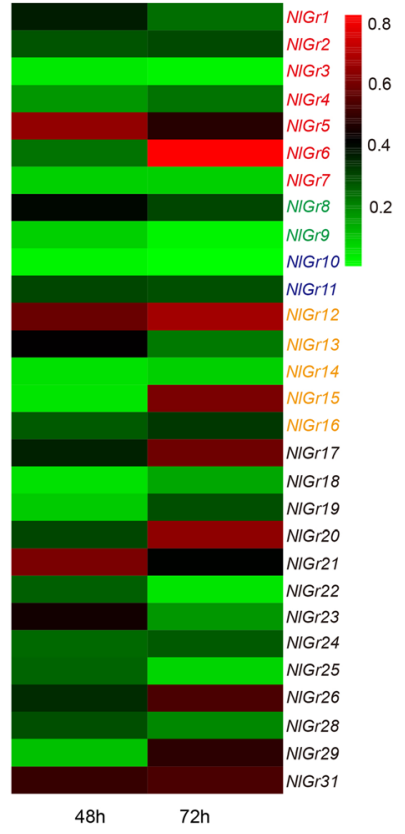

B

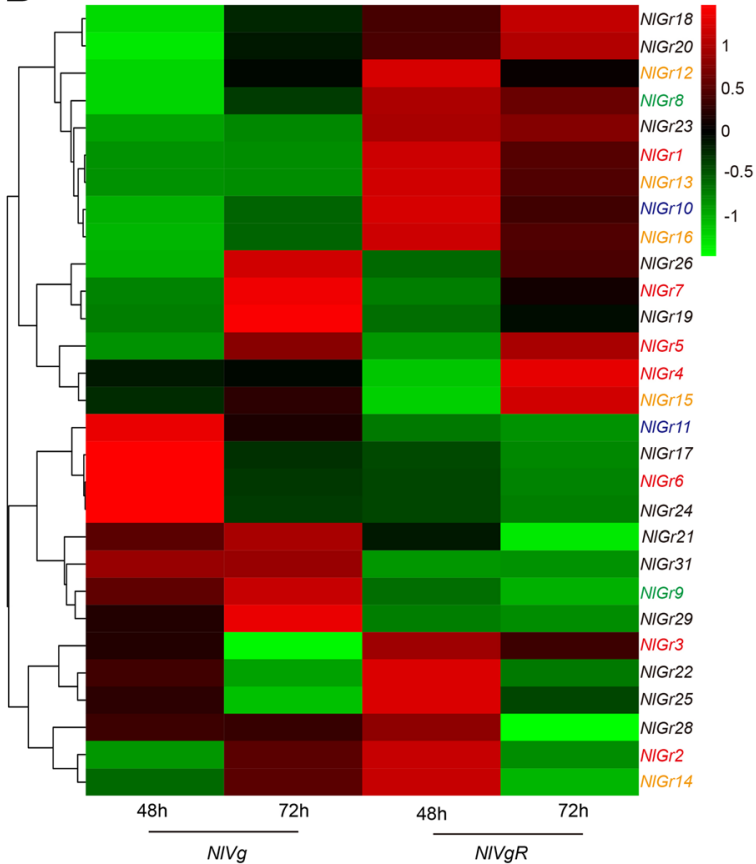

Fig. 6 Knockdown the expression of NIGrs in BPH and its effect on NIVg and NIVgR transcript accumulation. a: mRNA level of target genes; $\mathbf{b}$ : mRNA level of vitellogenin $(\mathrm{Vg})$ and vitellogenin receptor $(\mathrm{VgR})$ was detected by qRT-PCR; mRNA levels of females treated with dsGFP were used as controls. Different color fonts represent different predicted subfamilies: red: $\mathrm{CO}_{2}$ receptor subfamily; green: Gr43a-like receptor subfamily; blue: sugar receptor subfamily; orange: bitter receptor subfamily; black: unknown functions receptor. All mRNA levels are normalized by the $\beta$-actin mRNA levels and data are shown as the mean $(n=3)$

It is similar to the observations in Nasonia vitripennis whose Grs are most likely expressed in diverse chemosensory tissues, such as mouthparts, legs, and the ovipositor, as well as the digestive tract [33]. In other insects, such as $T$. castaneum, the Grs are mainly expressed in the labium and maxillae, femur, tibia, and tarsus of the adult prolegs [34]. In Aedge aegypti, the gustatory sensilla are located in stereotyped positions on the labella and tarsi of the legs [35], and there is also specific expression of $\mathrm{Grs}$ in these tissues [36]. $\mathrm{DmGr}$

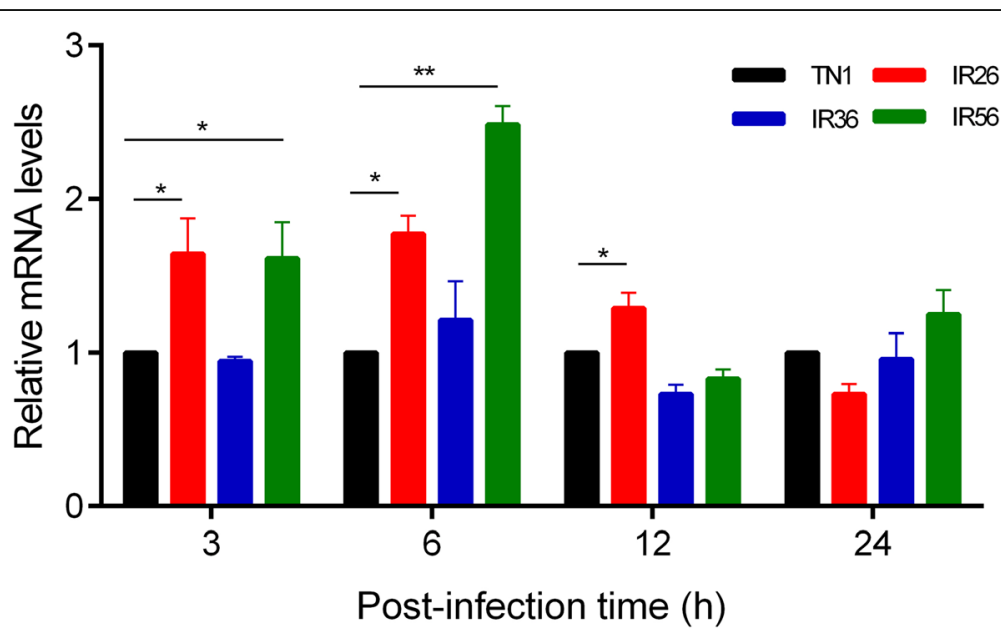

Fig. 7 Expression of N/Gr25 induced by various rice cultivars. N/Gr25 mRNA abundance was measured by quantitative real-time PCR. Total RNA was extracted from BPH at 3, 6, 12 and $24 \mathrm{~h}$ after feeding from different rice cultivars. TN1: Taichung Native 1, a BPH susceptible variety; IR36: International Rice 36, a BPH-resistant variety contains resistance gene bph2; IR56: International Rice 56, a BPH-resistant variety contains resistance gene Bph3. Data are shown as the means \pm SE of three replicates. Significant differences between two treatments are marked with an asterisk (t-test), * , at the 0.05 level; **, at the 0.01 level 
genes are found in abdominal tissues and the taste organs, including the labellum, pharyngeal organs, and tarsi $[37,38]$. Since BPH is a piercing sucking insect, the midgut is the main tissue used to digest food, absorb nutrients and remove toxic substances. NlGr25 was most highly expressed in the midgut and in 3th day female adults, we propose that that it might affect behaviors of $\mathrm{BPH}$ by responding to metabolite(s) in rice. Similarly, 12 DmGrs were expressed in midgut enteroendocrine cells, implying that the Grs have chemosensory roles in the intestine to regulate physiological functions, such as food uptake, nutrient absorption, or sugar homeostasis [38]. Some DmGrs have a higher mRNA level in the nymph stage, indicated that they play a role in the development of insects, as previously reported in Drosophila [39].

Alternative splicing is increasingly of interest in the study of evolution and adaptation of insects, as it provides the means for rapid functional innovations via very economical genomic changes [20]. However, to date, alternative splicing phenomenon has not been well studied for gustatory receptor family. In D. melanogaster, only three Gr genes (Gr23a, Gr39a and Gr28b) were found to have alternative splicing events [7], and alternative splicing in three genes brings the total number of Grs from 71 to 81 in Drosophila suzukii [40]. In BPH, 10 of 32 NlGrs have alternative splicing events, a higher percentage than that in Drosophila, which may be caused by the factor of the low number of $G r$ genes. Insect Grs often have a seven transmembrane structure [4], and the alternative splicing can change the transmembrane structure or the number units spanning the membrane and may greatly alter the function of the GR protein. The DmGr39a gene produces four isoforms through alternative splicing of various $5^{\prime}$-most exons [7]. The seventh transmembrane domain and $\mathrm{C}$-terminus are encoded by the last three exons, which are shared by the four isoforms, while the N-terminus and the first six transmembrane domains, which are unique to each isoform, are encoded by four alternative $5^{\prime}$ exons [21]. The RNAi of DmGr39a reduced courtship levels toward females in Drosophila, whilst it is not clear whether each isoform has a different function [21]. NlGr10 showed alternative first exon events, and the two isoforms both exhibited an entire seventh transmembrane domain. However, these two isoforms responded to different sugars (unpublished data). As a Gr43a-like receptor, NlGr8 was verified to have as many as ten splicing forms. Its homologous gene $D m G r 43 a$ plays a critical role in sensing internal fructose levels in the fly brain and is both necessary and sufficient to sense hemolymph fructose and promote feeding in hungry flies, while suppresses feeding in satiated flies [41]. Thus, we propose that $N l G r 8$ may play important roles in recognizing carbohydrates in rice phloem juice.
Gustatory receptors can affect insect behavior, such as feeding, oviposition or mating, by recognizing non-volatile plant secondary substances [3, 42]. The butterfly, Papilio xuthus, uses a gustatory receptor PxutGr1 to select its host during oviposition [42]. DmGr33a is required for proper oviposition to avoid ovicidal coumarin-laced food in D. melanogaster [3]. DmGr66a in the ventral cibarial sensory organ (VCSO) mediates the egg-laying attraction to lobeline and in neurons on the legs mediates positional aversion [43]. Functional and behavioral studies showed that other $\mathrm{Gr}$ genes (Dm8a, $D m 93 \mathrm{a}, D m 98 \mathrm{~b})$ responded to bitter compounds to induce avoidance responses, such as caffeine, umbelliferone, L-canavanine $[28,44]$. Our results showed that $N l G r$ can be induced by resistant rice varieties, suggesting that they may recognize some resistant or toxic metabolites in resistant rice, and subsequently affect BPH feeding behavior and oviposition behavior. And our previous study confirmed that NlGr11, a sugar receptor, accelerated the fecundity of $\mathrm{BPH}$ through the AMPK- and AKT-mediated signaling pathways [45].

\section{Conclusion}

In conclusion, we discovered that $N$. lugens has $32 \mathrm{Gr}$ genes, most of which belong to the bitter receptor and an unknown function clade. Ten of these $\mathrm{Gr}$ genes have two or more alternative splicing variants. The RNAi results showed that $27 \mathrm{NlGrs}$ are required for normal expression of fecundity markers. These findings contribute to the understanding of the interactions between $\mathrm{BPH}$ and rice.

\section{Methods}

\section{Insects}

A $N$. lugens laboratory strain was originally obtained from Guangdong Academy of Agricultural Sciences (GDAAS; Guangdong, China), and this strain was reared in a continuous laboratory culture on $\mathrm{BPH}$-susceptible rice plants (Huang Hua Zhan, bought from GDAAS). The insects were maintained in the laboratory at $26 \pm 2^{\circ}$ $C$ with $80 \pm 10 \%$ humidity and a light-dark cycle of L16:D8h.

\section{Sample preparation}

A total of 100 3-day-old brachypterous females were used for tissue dissection. The following tissues were used for RNAseq: head, midgut and legs. The fat body and ovary were also used for the gene expression profiling. Different development stages of $\mathrm{BPH}$, from the first nymph to five days after emergence, were collected. Total RNA was isolated using the TRIzol method (Invitrogen, Carlsbad, CA, USA) according to the manufacturer's protocol. The samples were treated with DNase, and their RNA contents were quantified using a 
microvolume spectrophotometer (NanoDrop

2000 , Thermo Fisher Scientific, Waltham, MA, USA).

\section{DGE analysis}

The cDNA libraries were constructed and sequenced by the Beijing Genomics Institute (BGI, Shenzhen, China) on the Illumina sequencing platform ( $\left.\mathrm{HiSeq}^{\mathrm{TM}} 2000\right)$, producing 100-bp pair-end reads (SRA accession: PRJNA504931). All clean reads were aligned to the reference transcript (generated in our laboratory, unpublished) of $N$. lugens using TopHat [46].

\section{Gene annotation}

We used three methods to identify the BPH gustatory receptor by assembled transcripts. Firstly, we download all the insect gustatory receptor gene family from $\mathrm{Na}$ tional Center for Biotechnology Information (https:// www.ncbi.nlm.nih.gov/) as a database, and the basic local alignment was used to search the DGE data of head, midgut and legs, respectively. Then tBLASTn searches against the NCBI database to confirm that the obtained sequences belong to the chemosensory family. In addition, the secondary structure model of the gustatory receptor by pfam (http://pfam.xfam.org/) and HMMER v3.1b2 (http://www.hmmer.org/) were used to search the target sequences in the protein database translated from the DEG database. Finally, using the gustatory receptor $\mathrm{C}$-terminal conserved sequences (hh(G/A/S) (A/S)hhTYhhhhhQF) as a template, tBLASTn searches were performed in DGE data. Using these three methods in combination, we obtained all of the gustatory receptors in $\mathrm{BPH}$.

\section{Cloning of NIGr genes}

The cDNA from various tissues, including head, midgut, fat body, ovary and whole body, was used as a template to clone the cDNA sequence of NlGrs. 3' RACE and 5' RACE PCR was further performed to get the complete cDNA sequences of Grs using a SMARTer RACE 5'/3' kit (Takara Bio) according to the manufactures' manuals. PCR products were purified using a Gel Extraction Kit (OMEGA Bio-Tek, USA), cloned into the $p E A S Y^{\circ}$ Blunt vector (TRANSGEN BIOTECH, Beijing, China) and then sequenced by IGE Biotechnology, Ltd. (Guangzhou, China). Then tBLASTn searches against the NCBI database to confirm that the cloning sequences belong to the Gr family. The primers used for gene clone are listed in supplementary file (see Additional file 5: Table S5).

\section{Phylogenetic analysis}

To investigate the expanding types of $29 \mathrm{BPH}$ Grs, we selected 266 published insect Grs as the reference source. Among these 266 Grs, 68 were from Drosophila melanogaster (DmGrs), 65 were from Bombyx mori
(BmGrs), 13 were from Apis mellifera (AmGrs), 58 were from Aedes aegypti (AgGrs), and 62 were from Tribolium castaneum (TcasGrs). Amino acid sequences were used for phylogenetic analysis in MEGA 6.0 [47]. A maximum-likelihood tree was calculated using the default settings and the Jones-Taylor-Tornton (JTT) with Freqs. (+F) model with partial deletions and 2000 bootstrap replications was applied in the calculation. The software TMpred (https://embnet.vital-it.ch/software/ TMPRED_form.html), HMMTOP (http://www.enzim.hu/ hmmtop/), and TMHMM (http://www.cbs.dtu.dk/services/ TMHMM/) were used for the prediction of transmembrane domains.

\section{Expression profiles of $\mathrm{NIGr}$ genes}

Total RNA was collected from the ovaries, midgut, fat body, legs and heads of third-day brachypterous female adults for tissue-specific expression profiles. RNA from the first to fifth instar nymph stages and brachypterous female adults (1-5 days old) were isolated for developmental expression profiles. The total RNA was extracted based on the method described above. For the gene transcription assay, $1 \mu \mathrm{g}$ RNA was used for first-strand cDNA synthesis using a PrimeScript ${ }^{\mathrm{Tm}} 1$ st Strand cDNA Synthesis Kit (Takara Bio, Kyoto, Japan). The mRNA level was detected by quantitative real-time PCR (qRT-PCR), the primers are listed in supplementary file (see Additional file 5: Table S5).

\section{Prediction and verification of alternative splicing}

To identify alternative splicing events in $\mathrm{BPH}$, we generated a relatively complete set of transcripts based on all available transcriptomes with different developmental stages, sexes and phenotypes (see Additional file 6: Table S6), following a computational pipeline presented in supplementary file (see Additional file 7: Figure S1). Firstly, the reads of each RNA-seq dataset were mapped separately to the reference genome using TopHat [46]. The alignment files produced by TopHat were loaded into Cufflinks to generate a transcriptome assembly for each dataset [48]. These assembled transcripts were combined into a general transcriptome assembly using the Cuffmerge module, and the alternative splicing events were identified according to the GTF file from Cuffmerge. Considering the high false positives of the alternative splicing events predicted from single-exon genes, we removed transcripts with single exon. After identification, cDNA was used as the template to clone the predicted isoforms sequence, and then cloned the NlGrs into the genome. The primers used for verification are listed in supplementary file (see Additional file 8: Table S7). 


\section{Herbivory treatments}

The resistant rice varieties (IR26, IR36, IR56) and susceptible rice variety TN1 (Taichuang native 1) (used as a control) were provided by the International Rice Research Institute (IRRI, Los Banos, the Philippines). Thirty one-day old brachypterous BPH females starved for at least $3 \mathrm{~h}$ prior to the experiment were put on each rice variety. Five individuals were sampled for qRT-PCR at each time point of $3 \mathrm{~h}, 6 \mathrm{~h}, 24 \mathrm{~h}$ and $24 \mathrm{~h}$ post feeding on rice. There were three replications for each treatment and sampling time point. All the collected samples were placed in liquid nitrogen immediately, and then stored at $-80^{\circ} \mathrm{C}$ for qRT-PCR.

\section{RNAi experiment and quantitative real-time PCR analysis} The dsRNA was produced using the T7 RiboMAX ${ }^{\mathrm{m}} \mathrm{Ex}-$ press RNAi System (Promega, USA). For each $N l G r$, thirty newly emerged virgin females were used for the injection experiment. 50-nanoliter of dsRNA $(4 \mu \mathrm{g} / \mu \mathrm{l})$ was injected into the side of the abdomen of BPH using 3.5 Drummond needles and the NARISHIGE IM-31 (Nikon, Tokyo, Japan). Five individuals that survived for 48 and $72 \mathrm{~h}$ after injection were randomly collected for RNA extraction. GFP gene (DQ389577) was used as a control dsRNA. Each treatment was performed in triplicate. qRT-PCR also used a previously described method [49]. The primers used for dsRNA preparation and real-time PCR are listed in Table S5.

\section{Statistical analysis}

For statistical analysis of the qRT-PCR results, the relative expression were calculated by $2^{-\Delta \Delta \mathrm{Ct}}$ values as previously described [50]. The mRNA levels of target genes were normalized relative to the $\beta$-actin mRNA levels, and all of the results are expressed as the means + SE; the differences between two groups were analyzed using $t$-tests. The differences between multiple groups were analyzed using one-way analysis of variance followed by Duncan's multiple range test for multiple comparisons. Differences were considered to be significant at $P<0.05$ and to be very significant at $P<0.01$.

\section{Additional files}

Additional file 1: Table S1. Summary of Nilaparvata lugens

transcriptome assembly. (XLSX 8 kb)

Additional file 2: Table S2. Screening for putative gustatory receptors of Nilaparvata lugens by tBLASTn. (XLSX $10 \mathrm{~kb}$ )

Additional file 3: Table S3. Prediction of potential gustatory receptors of Nilaparvata lugens by HMMER. (XLSX $9 \mathrm{~kb}$ )

Additional file 4: Table S4. Knockdown the expression of NIGrs in $\mathrm{BPH}$ and its effect on NIVg and NIVgR transcript accumulation. (XLSX $12 \mathrm{~kb}$ )

Additional file 5: Table S5. The primers used in this study. (XLSX $11 \mathrm{~kb}$ )
Additional file 6: Table S6. The data source for alternative splicing prediction. (XLSX $10 \mathrm{~kb}$ )

Additional file 7: Figure S1. Computational pipeline for identifying alternative splicing events in N. lugens from RNA-seq data. (JPG 64 kb)

Additional file 8: Table S7. The primers used in alternative splicing verification. (XLSX $9 \mathrm{~kb}$ )

\section{Abbreviations}

AKT: Protein kinase B; AMPK: Adenosine 5'-monophosphate (AMP)-activated protein kinase; BPH: The brown planthopper; cDNA: Complementary DNA; DGE: Digital gene expression; dsRNA: Double-stranded RNA; Gr: Gustatory receptor; GTF: Gene transfer format; JTT: Jones-Taylor-Tornton; NCBI: National Center for Biotechnology Information; Or: Odorant receptor; ORF: Open reading frame; PCR: Polymerase chain reaction; qRT-PCR: Quantitative real time RT-PCR; RACE: Rapid-amplification of cDNA ends; RNAi: RNA interference; SE: Standard error; tBLASTn: Translated basic local alignment search tool; Vg: Vitellogenin; VgR: Vitellogenin receptor

\section{Acknowledgements}

We thank Dr. Wei-Wen Chen, Dr. Yi Dong and Master's student Yong-Jin Cai (State Key Laboratory of Biocontrol and School of Life Sciences, Sun Yat-sen University) for their help in the RNA interference.

\section{Funding}

This work was funded by the National Natural Science Foundation of China (U1401212), the main support in the design of the study and collection, analysis, and interpretation of data and in writing the manuscript, and the China Postdoctoral Science Foundation (2017 M612808), the support in RNAi experiment and quantitative real-time PCR analysis.

\section{Availability of data and materials}

Raw data for this project have been submitted to the Sequence Read Archive (SRA) database of the National Center for Biotechnology Information (Accession number: PRJNA504931), the SRA records will be accessible with the following link: https://www.ncbi.nlm.nih.gov/bioproject/PRJNA504931/. The phylogenetic data was shown in TreeBASE database (Submission ID: 23701) with the following accession URL: http://purl.org/phylo/treebase/ phylows/study/TB2:S23701

\section{Authors' contributions}

WQZ made substantial contributions to conception and design, and given final approval of the version to be published. RP, LY, WWZ and ZRZ analyzed and interpreted the sequence data. Verification of selected genes and expression profiles investigation were performed by PY and LEC, with contributions from KK. Alternative splicing analysis was performed by KK and PY. Phylogenetic analysis and RNAi analysis was performed by KK, PY and LEC. KK drafted the manuscript and given final approval of the version to be published. WWZ and ZRZ were involved in drafting the manuscript for important intellectual content. All authors read and approved the final manuscript.

Ethics approval and consent to participate

Not applicable.

Consent for publication

Not applicable.

Competing interests

The authors declare that they have no competing interests.

\section{Publisher's Note}

Springer Nature remains neutral with regard to jurisdictional claims in published maps and institutional affiliations.

\section{Author details}

'State Key Laboratory of Biocontrol and School of Life Sciences, Sun Yat-sen University, Guangzhou 510275, Guangdong, China. ${ }^{2}$ State Key Laboratory of Rice Biology, Institute of Insect Sciences, Zhejiang University, Hangzhou 310029, Zhejiang, China. 
Received: 4 August 2018 Accepted: 18 December 2018

\section{Published online: 27 December 2018}

\section{References}

1. Hallem EA, Dahanukar A, Carlson JR. Insect odor and taste receptors. Annu Rev Entomol. 2006;51(10):113-35.

2. Engsontia P, Sanderson AP, Cobb M, Walden KKO, Robertson HM, Brown S The red flour beetle's large nose: an expanded odorant receptor gene family in Tribolium castaneum. Insect Biochem Mol Biol. 2008;38(4):387-97.

3. Poudel S, Lee Y. Gustatory receptors required for avoiding the toxic compound coumarin in Drosophila melanogaster. Mol Cells. 2016;39(4):310-5.

4. Clyne PJ, Warr CG, Carlson JR. Candidate taste receptors in Drosophila. Science. 2000;287(5459):1830-4.

5. Dunipace L, Meister S, McNealy C, Amrein H. Spatially restricted expression of candidate taste receptors in the Drosophila gustatory system. Curr Biol. 2001;11(11):822-35.

6. Scott K, Brady R, Cravchik A, Morozov P, Rzhetsky A, Zuker C, Axel R. A chemosensory gene family encoding candidate gustatory and olfactory receptors in Drosophila. Cell. 2001;104(5):661-73.

7. Robertson HM, Warr CG, Carlson JR. Molecular evolution of the insect chemoreceptor gene superfamily in Drosophila melanogaster. Proc Natl Acad Sci U S A. 2003;100:14537-42.

8. Bray S, Amrein H. A putative Drosophila pheromone receptor expressed in male-specific taste neurons is required for efficient courtship. Neuron. 2003; 39(6):1019-29.

9. Chyb S, Dahanukar A, Wickens A, Carlson JR. Drosophila Gr5a encodes a taste receptor tuned to trehalose. Proc Natl Acad Sci U S A. 2003;100:14526-30.

10. Thorne N, Chromey C, Bray S, Amrein H. Taste perception and coding in Drosophila. Curr Biol. 2004;14(12):1065-79.

11. Moon SJ, Koettgen M, Jiao Y, Xu H, Montell C. A taste receptor required for the caffeine response in vivo. Curr Biol. 2006;16(18):1812-7.

12. Jones WD, Cayirlioglu P, Kadow IG, Vosshall LB. Two chemosensory receptors together mediate carbon dioxide detection in Drosophila. Nature. 2007:445(7123):86-90.

13. Sato K, Tanaka K, Touhara K. Sugar-regulated cation channel formed by an insect gustatory receptor. Proc Natl Acad Sci U S A. 2011;108(28):11680-5.

14. Hill CA, Fox AN, Pitts RJ, Kent LB, Tan PL, Chrystal MA, Cravchik A, Collins FH, Robertson HM, Zwiebel LJ. G protein coupled receptors in Anopheles gambiae. Science. 2002;298(5591):176-8

15. Kent $L B$, Walden $K K O$, Robertson $H M$. The gr family of candidate gustatory and olfactory receptors in the yellow-fever mosquito Aedes aegypti. Chem Senses. 2008:33(1):79-93.

16. Richards S, Gibbs RA, Weinstock GM, Brown SJ, Denell R, Beeman RW, Gibbs R, Bucher G, Friedrich M, Grimmelikhuijzen CJP, et al. The genome of the model beetle and pest Tribolium castaneum. Nature. 2008;452(7190):949-55.

17. Wanner KW, Robertson HM. The gustatory receptor family in the silkworm moth Bombyx mori is characterized by a large expansion of a single lineage of putative bitter receptors. Insect Mol Biol. 2008;17(6):621-9.

18. Xu W, Papanicolaou A, Zhang HJ, Anderson A. Expansion of a bitter taste receptor family in a polyphagous insect herbivore. Sci Rep. 2016;6:23666.

19. Nilsen TW, Graveley BR. Expansion of the eukaryotic proteome by alternative splicing. Nature. 2010;463(7280):457-63.

20. Gibilisco L, Zhou Q, Mahajan S, Bachtrog D. Alternative splicing within and between Drosophila species, sexes, tissues, and developmental stages. PLoS Genet. 2016:12(12):e1006464.

21. Watanabe K, Toba G, Koganezawa M, Yamamoto D. Gr39a, a highly diversified gustatory receptor in Drosophila, has a role in sexual behavior. Behav Genet. 2011;41(5):746-53.

22. Ni L, Bronk P, Chang EC, Lowell AM, Flam JO, Panzano VC, Theobald DL, Griffith LC, Garrity PA. A gustatory receptor paralogue controls rapid warmth avoidance in Drosophila. Nature. 2013;500(7464):580-4.

23. Qiu J, He Y, Zhang JQ, Kang K, Li TC, Zhang WQ. Discovery and functional identification of fecundity-related genes in the brown planthopper by largescale RNA interference. Insect Mol Biol. 2016:25(6):724-33.

24. Zhai YF, Sun ZX, Zhang JQ, Kang K, Chen J, Zhang WQ. Activation of the TOR signalling pathway by glutamine regulates insect fecundity. Sci Rep. 2015;5:10694.

25. Xue J, Zhou X, Zhang C-X, Yu LL, Fan HW, Wang Z, Xu HJ, Xi Y, Zhu ZR, Zhou WW, et al. Genomes of the rice pest brown planthopper and its endosymbionts reveal complex complementary contributions for host adaptation. Genome Biol. 2014;15(12):521.
26. Dahanukar A, Foster K, van Naters W, Carlson JR. A gr receptor is required for response to the sugar trehalose in taste neurons of Drosophila. Nat Neurosci. 2001;4(12):1182-6.

27. Slone J, Daniels J, Amrein H. Sugar receptors in Drosophila. Curr Biol. 2007; 17(20):1809-16.

28. Lee Y, Kang MJ, Shim J, Cheong CU, Moon SJ, Montell C. Gustatory receptors required for avoiding the insecticide L-Canavanine. J NeurosC. 2012;32(4):1429-35.

29. Shim J, Lee Y, Jeong YT, Kim Y, Lee MG, Montell C, Moon SJ. The full repertoire of Drosophila gustatory receptors for detecting an aversive compound. Nat Commun. 2015:6:8867.

30. Robertson HM, Wanner KW. The chemoreceptor superfamily in the honey bee, Apis mellifera. expansion of the odorant, but not gustatory, receptor family. Genome Res. 2006;16(11):1395-403.

31. Smadja C, Shi P, Butlin RK, Robertson HM. Large gene family expansions and adaptive evolution for odorant and gustatory receptors in the pea aphid, Acyrthosiphon pisum. Mol Biol Evol. 2009;26(9):2073-86.

32. McBride CS, Arguello JR. Five Drosophila genomes reveal nonneutral evolution and the signature of host specialization in the chemoreceptor superfamily. Genetics. 2007;177(3):1395-416.

33. Robertson HM, Gadau J, Wanner KW. The insect chemoreceptor superfamily of the parasitoid jewel wasp Nasonia vitripennis. Insect Mol Biol. 2010;19:121-36.

34. Abdel-Latief M. A family of chemoreceptors in Tribolium castaneum (Tenebrionidae: Coleoptera). PLoS One. 2007;2(12):e1319.

35. Lee RM, Craig DA. Fine structure of the sense organs on the labella and labium of the mosquito Aedes aegypti (L). Open Entomol J. 2009:3(1):7-17.

36. Sparks JT, Vinyard BT, Dickens JC. Gustatory receptor expression in the labella and tarsi of Aedes aegypti. Insect Biochem Mol Biol. 2013;43(12):1161-71.

37. Thorne N, Amrein H. Atypical expression of Drosophila gustatory receptor genes in sensory and central neurons. J Comp Neurol. 2008;506(4):548-68.

38. Park JH, Kwon JY. A systematic analysis of Drosophila gustatory receptor gene expression in abdominal neurons which project to the central nervous system. Mol Cells. 2011;32(4):375-81.

39. Saina M, Busengdal $H$, Sinigaglia $C$, Petrone $L$, Oliveri $P$, Rentzsch F, Benton R. A cnidarian homologue of an insect gustatory receptor functions in developmental body patterning. Nat Commun. 2015;6:6243.

40. Hickner PV, Rivaldi CL, Johnson CM, Siddappaji M, Raster GJ, Syed Z. The making of a pest: insights from the evolution of chemosensory receptor families in a pestiferous and invasive fly, Drosophila suzukii. BMC Genomics. 2016;17(1):648.

41. Miyamoto $T$, Slone J, Song $X$, Amrein $H$. A fructose receptor functions as a nutrient sensor in the Drosophila brain. Cell. 2012;151(5):1113-25.

42. Ozaki K, Ryuda M, Yamada A, Utoguchi A, Ishimoto H, Calas D, Marion-Poll $\mathrm{F}$, Tanimura T, Yoshikawa $\mathrm{H}$. A gustatory receptor involved in host plant recognition for oviposition of a swallowtail butterfly. Nat Commun. 2011;2: 542.

43. Joseph RM, Heberlein U. Tissue-specific activation of a single gustatory receptor produces opposing behavioral responses in Drosophila. Genetics. 2012;192(2):521-32.

44. Poudel S, Kim Y, Kim YT, Lee Y. Gustatory receptors required for sensing umbelliferone in Drosophila melanogaster. Insect Biochem Mol Biol. 2015;66: $110-8$.

45. Chen WW, Kang K, Yang P, Zhang WQ. Identification of a sugar gustatory receptor and its effect on fecundity of the brown planthopper Nilaparvato lugens. Insect Sci. 2017. https://doi.org/10.1111/1744-7917.12562.

46. Trapnell C, Pachter L, Salzberg SL. TopHat: discovering splice junctions with RNA-Seq. Bioinformatics. 2009;25(9):1105-11.

47. Tamura K, Stecher G, Peterson D, Filipski A, Kumar S. MEGA6: molecular evolutionary genetics analysis version 6.0. Mol Biol Evol. 2013;30(12):2725-9.

48. Trapnell C, Roberts A, Goff L, Pertea G, Kim D, Kelley DR, Pimentel H, Salzberg SL, Rinn JL, Pachter L. Differential gene and transcript expression analysis of RNA-seq experiments with TopHat and cufflinks. Nat Protocols. 2012;7(3):562-78.

49. Kang K, Yang P, Pang R, Yue L, Zhang WQ. Cycle affects imidacloprid efficiency by mediating cytochrome $\mathrm{P} 450$ expression in the brown planthopper Nilaparvata lugens. Insect Mol Biol. 2017;26(5):522-9.

50. Livak KJ, Schmittgen TD. Analysis of relative gene expression data using realtime quantitative $P C R$ and the $2^{-\Delta \Delta C T}$ method. Methods. 2001:25(4):402-8. 